\title{
El ejercicio de la parentalidad en la clínica con adolescentes: una propuesta de intervención psicoanalítica
}

Daniela Fernández Olguin*1

Mediante la revisión de un caso clínico se busca analizar las configuraciones del ejercicio de la parentalidad en la clínica con adolescentes desde una perspectiva psicoanalitica con el fin de proponer su integración y análisis en este contexto. Se propone que este ejercicio no sólo implicaría transformaciones psíquicas para los adolescentes, sino también para los padres, quienes están implicados por su propia historia.

Palabras claves: Adolescencia, parentalidad, psicología clínica, psicoanálisis

${ }^{* 1}$ Universidad de Viña del Mar (Viña del Mar, Chile) 


\section{Introducción}

El presente artículo utiliza la perspectiva psicoanalítica como marco teórico de referencia y análisis respecto al estudio del ejercicio de la parentalidad y sus posibles aportes a la clínica con adolescentes mediante la presentación de un caso clínico en el marco de un proceso psicoterapéutico de orientación psicoanalítica. En función de esto, se expondrán algunos antecedentes teóricos en torno al ejercicio de la parentalidad durante la adolescencia con el fin de circunscribir la comprensión de estos conceptos. Posteriormente, se expondrán 452 viñetas de un caso clínico en el cual se trabajó con un adolescente y sus padres, para posteriormente analizar y reflexionar las implicancias del ejercicio de la parentalidad en la clínica con adolescentes, así como su aporte en la intervención clínica con adolescentes.

A partir de los años ochenta y noventa las funciones y roles de los padres comienzan a reagruparse bajo un nuevo concepto: la parentalidad (Houzel, 2004). El concepto de parentalidad intenta subrayar: “(...) que no es suficiente el procrear ni el ser designado como padre para llenar todas las condiciones, sino que es necesario "convertirse en padre", lo que se logra a través de un proceso complejo que implica los niveles conscientes e inconscientes del funcionamiento mental" (Houzel, 2004, p. 27). Así, se entiende como un proceso en el cual se impacta el psiquismo de los padres en su proceso de parentalización, y también el psiquismo del hijo e hija. La parentalidad puede ser definida como el ejercicio que "se conforma de representaciones mentales, afectos, deseos y comportamientos de los padres en relación con su hijo (...)" (Mazet, 2004, p. 201). Es una labor de construcción (Wettengel, 2009), que hace referencia al sostén afectivo y efectivo de la crianza, a la transmisión de la cultura, los deseos, miedos y fantasmas, y el índice libidinal para que esto emerja. Esta función 


\section{ARTIGOS}

generará un proceso de transformación tanto en hijos como en adultos, ya que habrá un antes y un después de convertirse en padre.

Dentro de la complejidad de estas funciones Houzel (2004) describe como un eje relevante el ejercicio de la parentalidad, el cual da cuenta de todos los aspectos relacionados con la transmisión simbólica que opera en la familia. Se podría referir que mediante el ejercicio de la parentalidad se anuda el psiquismo con lo cultural en una relación recíproca donde ambos van inscribiéndose: "De hecho, la investigación psicoanalítica ha demostrado que la organización de la psique individual no puede ser entendido sin referencia al grupo en que se desarrolla y su estructura íntima hace eco de la estructura social en la que el individuo está inmerso" (Houzel, 2004, p. 127).

Esto se relaciona a lo que Gutton (1993) plantea como el valor estructurante del Edipo en la adolescencia, el que remite a una reviviscencia del proceso acaecido en la infancia en la cual el niño debe construir una relación sólida con sus padres y posteriormente por la cultura. Durante el periodo edípico, el niño y la niña de forma diferencial desarrollan relaciones objetales que generen un movimiento desde el amor hacia las figuras parentales para lograr la exogamia y renuncia a estos objetos de amor primarios: “(...) y se libera así de la presencia necesaria del objeto: el objeto, siendo simbolizado, puede ser evocado (o asesinado) in absentia" (Marty, 2009, p. 94).

Ahora, debido a los cambios corporales del púber en su evolución sexual, las representaciones infantiles incestuosas son realizables, por lo que el incesto eventualmente sería posible. Sin embargo, Gutton (1993) diferencia al Edipo puberal como asimétrico, ya que si bien existe una investidura erótica del progenitor incestuoso, también hay una desinvestidura erótica del rival, la cual es facilitadora de su asesinato simbólico. Esto es lo que Freud (1905/2013) denomina la regeneración puberal del complejo de Edipo: la reactivación o revisitación edípica.

Con el fin de analizar los aportes de la integración del ejercicio de la parentalidad en una intervención clínica con adolescentes, se expondrá a modo de ilustración la viñeta de un caso clínico trabajado en el contexto de la atención particular con periodicidad de una sesión semanal durante 9 meses con interrupciones esporádicas por la salud del paciente (aproximadamente una inasistencia al mes). El trabajo incluyó entrevistas e intervenciones con el joven, la madre y el padre - individuales y en pareja —, de acuerdo a las emergencias que se iban desarrollando en el caso. Esto, con la precaución de no establecer un apoyo psicoterapéutico para la madre y el padre, sino un espacio en el cual el ejercicio parental pudiera ser analizado y abarcado dentro 
de su particularidad. Se elige este caso ya que expone elementos relevantes en el estudio del ejercicio de la parentalidad, así como el análisis de este aspecto, la cual permitió generar lineamientos particulares en el caso en cuestión.

\section{El caso de Antonio: \\ "Llegué a un punto en que le tengo miedo a mi propio hijo"1}

Antonio tiene 13 años y asiste a un colegio dentro de la ciudad de Santiago de Chile. Vive con su madre de 43 años, su padre de 42 años y su hermano mayor de 20 años, quien asiste a la universidad. Ambos padres trabajan jornada completa, siendo el tiempo de traslado hacia sus trabajos de hasta 1 hora de viaje. En la primera entrevista, la madre manifiesta preocupación por su hijo, debido a que hace 3 semanas no asiste al colegio ya que pasa 15 horas diarias utilizando videojuegos en línea, específicamente el juego "World of Warcraft". ${ }^{2}$ La madre agrega que debido a sus jornadas de juego extensas, Antonio ha tenido problemas en su postura y su médico decide instalarle un corsé por un período de un año. Respecto a esto, Antonio refiere que "le da lo mismo", sin embargo, en cuanto la fecha de la instalación del corsé se hace más cercana, la madre relata actitudes más violentas de parte de Antonio: en una ocasión, cuando la madre lo insta a que asista al colegio y lo despierta en la mañana, Antonio intenta golpearla. Esto ha impedido que el procedimiento se pueda llevar acabo.

La situación es descrita por el padre como insostenible y refiere que en un momento de "desesperación" — como lo define él — decide cortar la luz de toda la casa, con el fin de impedir que Antonio continúe jugando. Frente a esto, Antonio le grita y le lanza un vaso, el cual el padre debe esquivar. Luego, Antonio recoge uno de los pedazos rotos y corta su brazo: "Llegué a un punto que le tengo miedo a mi propio hijo: es como el mundo al revés", expresa el padre. Frente a esto, luego de conocer a Antonio en una sesión individual para escuchar su perspectiva de la situación, se realizan tres sesiones simultáneas

${ }^{1}$ Nombres y datos han sido modificados para mantener el anonimato y confidencialidad del caso.

${ }^{2}$ World of Warcraft (abreviado comúnmente como WoW) es un video juego de rol multijugador masivo en línea. Su argumento se sostiene en la exploración y combate de varios monstruos y jugadores, completando misiones e interactuando con personajes jugadores y no jugadores. 


\section{ARTIGOS}

con el padre, quien desarrolla ideas personales en torno a lo que comprende por "autoridad", significante que ha relacionado con el ser padre: "Recuerdo que a mi padre le tenía miedo, y pensaba que tenía que ser así. Siempre me impuse con ellos, pero con el Antonio no me está resultando, pienso que soy mal padre porque no le doy miedo a mi hijo... Y ahora que lo digo suena muy extraño esto". Esto, se relaciona con lo que Antonio despliega en la entrevista previa a las reuniones con el padre: "Me cansé de tenerle miedo, llevo mucho tiempo así y no soy feliz, pero parece que él es así y no hay nada que hacer. Me voy a tener que ir de la casa para que no me moleste más".

Luego de estas sesiones donde se aborda la posibilidad de construir nuevas significaciones sobre lo que es el ser padre y "la autoridad", se retoman las entrevistas con Antonio, quien inicialmente se muestra reacio a hablar, diciendo que la situación "le da lo mismo" y sólo explayándose más cuando se refiere a su padre, criticándolo. Frente a este diálogo repetitivo y muchas veces monosilábico, se utiliza el recurso del juego para comprender la visión de mundo de Antonio, proponiéndole realizar una maqueta del mundo que ha creado virtualmente. Responde positivamente, logrando establecer un rapport adecuado y desplegar de forma más fluida su discurso frente a la problemática: "Es que me da cosa el corsé, se van a burlar, no podré hacer nada y no podré jugar. Y tampoco podré contarle a nadie, porque nadie lo entiende si no lo usa".

Frente a este discurso que alude al desamparo en esta situación corporal provocada por el uso de videojuegos, nuevamente se realiza una entrevista con ambos padres, quienes manifiestan su preocupación por la poca mejora de Antonio, ya que aun no retoma su asistencia al colegio, y sobre todo la madre refiere su preocupación por la situación y progresivamente comienza a expresar de forma más directa en el contexto del dispositivo clínico sus miedos y frustración en torno a la problemática de Antonio, con un discurso inicialmente confuso respecto a sus sentimientos e ideas en torno a por qué no ha podido resolver por si misma esta situación. El padre inicialmente la secunda, para posteriormente retomar su preocupación por el miedo que le da su hijo: "A veces no me atrevo a decirle nada, ni cómo me siento, por qué no sé como va a reaccionar".

La angustia de la madre se vincula a su desconfianza del dispositivo terapéutico: "Pienso que usted como es psicóloga me va a retar", manifiesta en muchas ocasiones. Frente a esto, se entrevista a la madre de forma individual en dos ocasiones, en donde refiere de forma afectada las críticas que ha recibido durante la crianza de sus hijos, sobre todo de su propia madre, 


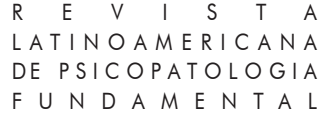

la abuela de Antonio: "Siempre sentí que lo hacía mal, que era tan difícil ser mamá, como mi mamá se quejaba tanto. Sentía que no podría y yo creo que eso me hace sentir mal con lo que le pasa al Antonio". Se elaboran estos aspectos transferenciales, proponiendo la historización de su propio lugar como hija, al cual acude al ejercer la maternalidad. El poner en discurso estas ansiedades facilita la transferencia hacia la psicoterapeuta por parte de la madre, quien da cuenta de interés y comprensión frente al proceso de Antonio, en vez de la demanda de soluciones inmediatas. Al mismo tiempo, se muestra mucho más cercana al padre de Antonio, logrando conversar respecto a la situación y no sólo "llorar y discutir".

Al retomar las sesiones con Antonio, muestra una mejor integración de sus afectos vinculados con la escuela y paulatinamente empieza a reintegrarse a esta: primero asiste un día a la semana, luego dos, y así hasta llegar a asistir cuatro de cinco días a la semana al colegio. Este proceso tiene un curso irregular, ya que en ocasiones Antonio pide a su madre llegar más tarde al colegio, frente a lo que la madre se opone y esto genera discusiones entre ambos, que no vuelven a llegar a la violencia física. Si bien se exponen las mejoras paulatinas de Antonio frente a su sintomatología, la madre cada 456 algunas semanas retorna a una posición de angustia y desesperanza respecto a su hijo, siendo un tema relevante en su discurso la comparación desde un modelo ideal lo que fue su adolescencia con el desarrollo adolescente de Antonio, lo cual parece frustrarla más e intensificar su malestar y angustia frente a las actitudes de Antonio, y adicionalmente, ante enfrentar las problemáticas concretas que implican la inasistencia parcial de su hijo al colegio debido a que continúan episodios que remiten a su adicción a los videojuegos, refiriendo sobre si misma incapacidad para solucionar en su "rol de madre" esta situación: "No creo haber sido una mala mamá, o quizás sí, siempre pensé que hacía lo correcto pero ahora dudo mucho".

En base a esto, se retoman las sesiones con ambos padres, en donde la madre relata: "Yo no era así cuando chica, no sé, como tan violenta, nunca pensé que como madre tendría que enfrentarme a la violencia de mi hijo... No estaba en mi parámetros, yo no era así con mis papás, no sé que pensarían los abuelos de Antonio si supiera cómo están las cosas entre nosotros". El padre de Antonio refiere conocer algo de estos problemas familiares, sólo que ahora se posiciona en un rol más activo frente al malestar de la madre de Antonio: "Era como que estábamos viendo como algo solo del Antonio y tiene también que ver con nosotros". 


\section{ARTIGOS}

Respecto a las actitudes violentas Antonio, quien inicialmente refiere que "no sabe" por qué reacciona así, con el paso de las sesiones, mediante entrevistas y acercamientos gráficos al mundo del video juego que utiliza, logra elaborar algunas respuestas respecto a su malestar: "Es raro estar solo todo el día, mi papá trabaja, mi mamá trabaja, mi hermano va a la universidad, ahora están más cerca pero yo creo que es por este problema". Los espacios de soledad plantean para Antonio la pérdida de referentes en torno a su vida y sobre todo su proceso puberal: "Me siento raro, no me gusta mi cuerpo, ahora con el corsé tampoco sé bien cómo me veré o qué haré, y no se si debo contarle a mi papá esto, porque no sé si me entenderá, al final exploto y por eso reviento con ella". Se le consulta por qué le preguntaría a su papá, a lo que responde: "Porque es hombre y supongo que debe entender lo que pasa... No sé".

Los últimos 4 meses de tratamiento la madre - mediante entrevistas mensuales en conjunto con el padre de Antonio - va elaborando algunos aspectos vinculados con la exigencia sobre el rol maternal, los cuales vincula con la relación con su padre, a quien define como "agresivo, violento, me daba miedo". Es posible ver que gran parte de la reacción que la madre tenía frente a los episodios de Antonio estaba mediada por su propia experiencia con la agresividad y los aspectos no elaborados de esta. La sensación de desamparo que describe inicialmente y que retorna en los episodios que en el tiempo van disminuyendo pero que permanecen en Antonio, logra ser comprendida a partir de la propia historia de la madre y la reactivación de conflictivas edipicas de su propia historia, que no permitía el poder posicionarse en un lugar de madre - y ya no hija temerosa — frente a la problemática de su hijo.

El padre por su parte pareciera identificarse con esta idea de lo paternal: "No quiero que me hijo me tenga miedo, quiero que confíe, al final no estaba dándome la oportunidad de ser el padre que quiero, sino el que pensé que había que ser". El repetir un lugar de la historia de él como hijo, así como de su esposa, pareciera haber generado un cuestionamiento respecto a su posición en la familia, así como a su lugar como hijo, refiriendo en momentos de forma emocionada: "comprendo porque su padre era así, como violento, pero yo no quiero eso".

Cercano a la finalización del tratamiento, los padres anuncian que en conjunto con Antonio eligieron otro tipo de tratamiento para su problema de postura, el cual consiste en terapia con kinesiólogos y fisioterapeutas, en vez del corsé. Esto, disminuye de manera notoria las reacciones agresivas de Antonio frente a los límites impuestos sobre horarios y uso del computador, 


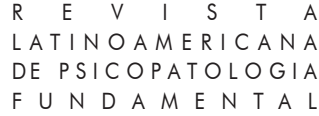

ya que si bien se generan discusiones sobre todo con su madre, estas no terminan en agresiones físicas ni verbales. Por otro lado, la madre comienza a reflexionar en torno a sus propios miedos en torno al ejercicio parental con Antonio, y cómo estos estaban vinculados con su historia como hija, la cual retornaba en la relación con su hijo. A medida que comienza a posicionarse como sujeto de su historia y reencontrarse en ella como madre, establece una nueva mirada respecto a su hijo y su problemática: "Culparme no sé si ayude en algo, o si lo ayude a él o a mi, pero si creo que tengo que estar más presente pero distinta, ya no soy mamá de un niño, sino de un joven". Este nuevo posicionamiento se condice con el mayor interés que Antonio muestra en relacionarse con sus pares, a medida que se reintegra al colegio, ahora cuatro días a la semana en jornada completa, hasta finalizar el año escolar con un promedio que le permite pasar de curso, lo cual facilita además la disminución del uso del videojuego, en tanto prioriza su tiempo libre en ver a sus pares.

\section{¿Cómo se configura el ejercicio de la parentalidad en la clínica con adolescentes?}

Revisando el caso a modo de ilustración, es posible observar por un lado el discurso de desamparo que en ocasiones los padres de adolescentes refieren, respecto a un desconocimiento de su hijo, así como de temores frente a cómo operar en su lugar de padres frente a las problemáticas acaecidas, sobre todo cuando implican conductas agresivas como en el caso presentado. Al mismo tiempo, la noción de desamparo puede escucharse en el discurso y conductas de Antonio, en relación a la elaboración no sólo de la impronta puberal propia de su etapa del desarrollo, sino además al artefacto del corsé que debía usar por un largo período de tiempo debido a la transformación de su postura por el uso de video juego, espacio virtual utilizado para buscar sostener y elaborar los sentimientos depresivos en torno al abandono de la infancia y la soledad del proceso (Marty, 2001). Estos procesos de elaboración psíquica, iniciados por lo que Gutton (1993) denomina como violencia puberal, se presentarían con la dificultad propia de estas eclosiones para un aparato psíquico que no puede sostener mantenerse como en la infancia y que requiere del sostén adulto, con la complejidad que esto implica. Es necesario que circule una nueva temporalidad psíquica que permita la simbolización de estos cambios puberales los cuales no se pueden sostener para el adolescente de forma individual, y siendo necesaria la integración de los padres en su ejercicio dentro 


\section{ARTIGOS}

del contexto clínico durante la adolescencia, lo que es cada vez más complejo en nuestro contexto socioeconómico e histórico actual. Los elementos de culpa que los padres - y sobre todo la madre - en ocasiones expresaba también se vinculaban al ser "trabajadores", estableciendo una carga mayor a su ejercicio, desde un discurso de déficit.

El posicionamiento que los padres tomen respecto a este resurgimiento arcaico y el sostén ofrecido propician la incorporación de aspectos culturales y sociales, tarea relevante en la adolescencia, vehiculizada inicialmente por las figuras parentales quienes dentro del ejercicio de la parentalidad transmiten estos elementos simbólicos: "La coexistencia en el tiempo, entre los hechos que rodean al complejo de Edipo y la socialización es coherente con la capacidad madurativa de niño que permite incorporar los conceptos culturales a la estructura psíquica" (Cuestas, 2009, p. 157).

A partir de la integración de los padres en el proceso psicoterapéutico de adolescentes es posible reflexionar sobre el lugar que se le ha otorgado a la habilitación del oficio de madre y padre, lo cual abre una temática que sesga la subjetividad parental, que tiene relación con el problema de la supuesta relación naturalizada con la ley simbólica, como si fuera algo ya dado y sin transformaciones, desde donde se podrían ejercer acciones que pautan, prescriben y proscriben los limites para el niño o adolescente (Wettengell, 2009).

Al mismo tiempo, las potenciales dificultades o fallas que los padres representen en su ejercicio, no corresponden sólo a una visión en la cual el sujeto es el único responsable. Todas las transformaciones sociales conllevarían un déficit en las operaciones de los padres, por lo cual se da cuenta de las dificultades propias y contextuales que pueden surgir en el ejercicio de la parentalidad en la adolescencia.

De este modo, el lugar de los padres en la adolescencia es más complejo que la repetición del Edipo infantil (Freud, 1905/2013; Freud, A., 1976; Dolto, 1981), ya que da cuenta de repercusiones a nivel psíquico que no sólo causan un efecto en el adolescente, sino que implican reelaboraciones propias para las figuras parentales. Esto no implica sólo las acciones específicas o concretas que los padres puedan realizar frente al accionar de su hijo adolescente, sino también las operaciones psíquicas que se configuran durante este periodo, las cuales no representan sólo una transmisión concreta por parte de los padres, sino que también invocan a su propia trabajo psíquico como sujetos, en este caso relacionados con la relación con el padre de la madre. La emergencia de su propio Complejo de Edipo en este proceso como una configuración particular y no sólo repetición, así como la vivencia psíquica particular que 
construyen en este proceso relacional con su hijo adolescente, da cuenta de una configuración particular de la parentalidad en este momento vital, el cual puede ser descrito inicialmente a partir de su propio Complejo de Edipo o Contra Edipo parental (Marty \& Missonnier, 2010).

\section{Discusiones}

La transmisión parental tiene relación con la inscripción que el sujeto realiza en su propia historia y filiación. Por esto, involucra directamente la propia historia de los padres, que debe configurarse ante la irrupción puberal y las elaboraciones de ésta. Las transmisiones parentales dan cuenta de la forma en que los propios padres han inscrito lo cultural por su Edipo, y cómo lo transmiten a sus propios hijos. Por esto, dentro del dispositivo clínico, los discursos de los padres frente a las conductas de sus hijos adolescentes van más allá de lo reactivo, sino que involucran configuraciones que se inscriben dentro de sus propias coordenadas históricas.

La parentalidad no hace efecto sólo en el sujeto hijo, sino que además en los sujetos que ejercen la función parental en su propia estructuración subjetiva. Integrar este aspecto en la intervención clínica con adolescentes, otorga mayor riqueza en el análisis de los elementos que van configurando el ejercicio parental, con las transmisiones y prácticas en el vínculo con los adolescentes.

El trabajo de la parentalidad en la adolescencia da cuenta de un quehacer adulto que no se acota a la niñez, sino que se desarrolla pensando en la demanda constante en torno a la transmisión cultural. En la adolescencia, el ejercicio de la parentalidad también puede pensarse desde la noción de apuntalamiento, reflexionando como la nueva sexualidad que se impone para el adolescente busca ser re-presentada, gracias a elementos culturales. Y al mismo tiempo, cómo esta imposición genera a su vez configuraciones particulares para los propios padres, los cuales al ser elaborados e interpretados en el contexto de la intervención con el adolescente propician un nuevo posicionamiento en quienes ejercen la parentalidad, con la diferenciación entre elementos de su historia y cómo estos se relacionan con su ejercicio parental, logrando mayor comprensión del tratamiento, así como compromiso, debido a que existe una mayor integración de su propia persona en el proceso psicoterapéutico del adolescente, explicitando la relevancia de su discurso en la comprensión de las problemáticas de su hijo. 


\section{ARTIGOS}

La integración del lugar de los padres considerando al Edipo como una representación de una relación particular en un plano simbólico, mediante la cual se busca la promoción de las condiciones de constitución subjetiva, no sólo como una lucha edípica, sino también como un proceso en los cuales se accionen recursos psíquicos para poder sostener al adolescente. Por esto, no es tanto los padres reales los que aparecen en escena como relevantes, sino más bien los sujetos que realicen esta función. Es necesario dar cuenta que la problemática de la parentalidad representa un conflicto a lo largo de la vida, no sólo como repetición o reedición, sino también como una configuración nueva a partir de su ejercicio o el lugar en el cual el sujeto se encuentra en esta relación tríadica o terciaria.

La lógica de la terceridad apela al ingreso en el psiquismo de otro, distinto, de la alteridad, que pareciera alterar lo "natural", y es a partir de la noción de la alteridad que el adolescente puede continuar relacionándose con la cultura, con la alteridad subjetiva. Por esto, la alteridad no irrumpe solamente como otro concreto, sino también como lo diferente, y lo que hace conflicto en el propio discurso y en el aparato psíquico. Entonces, es a través de elementos presentes en el psiquismo de la madre y el padre, en relación a sus propias figuras del pasado, como sus propios padres que irrumpen en este ejercicio, y que dan cuenta de que la cadena histórica de la parentalidad va más allá de la relación concreta padre-madre-hijo. La lógica tercera podría pensarse a su vez como la propia historia de los padres en sus ejercicios, como un proceso de historización que nunca se agota.

De este modo, la alteridad no implica sólo la pregunta por otro concreto o particular, sino también por la propia otredad que está en el psiquismo, por lo que irrumpe y hace conflicto, ya que problematiza la supuesta estructuración finalizada. De este modo, las operaciones parentales en la adolescencia, transmiten a su vez este conflicto, el del encuentro con el otro y lo otro del psiquismo parental, lo cual ambos autores conceptualizan como la presencia del propio Edipo de los padres en la configuración del Edipo adolescente.

La adolescencia surge de este modo como una construcción necesaria para poder pensar este proceso no como lineal, sino también desde el conflicto, noción siempre ha estado presente en la teoría psicoanalítica. Como propuesta, podría referirse que la adolescencia pareciera ser considerada como un síntoma, en tanto pone en juego un conflicto psíquico e intersubjetivo: psíquico para el propio adolescente, psíquico para los propios padres, en tanto la manifestación pulsional del adolescencia propicia la revivificación de las fuerzas pulsionales de los propios padres, no sólo de su propia adolescencia, 


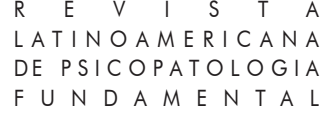

sino de su propia pulsionalidad como padres y adultos. Y, como conflicto intersubjetivo, retomando la idea freudiana del conflicto entre generaciones, pero considerando a su vez elementos culturales y sociales que se ven implicados en la consideración de las generaciones.

Por esto, no es sólo la metamorfosis de los adolescente, sus padres y la parentalidad, sino también la necesidad de plantear una metamorfosis en el ejercicio clínico con adolescentes y sus padres, que no signifique el replicar el método de trabajo con los adultos o los niños, ni tampoco la desvinculación de ambos actores en el proceso, sino en la construcción de orientaciones propias que vayan en la línea del contexto actual en el cual se da la parentalidad durante la adolescencia, integrando elementos históricos vinculados con los padres y su propia vivencia de la parentalidad como hijos, así como su propia adolescencia, puesta en historia frente al desarrollo

\section{Referencias}

Cuestas, F. (2009). Integración, exclusión social y adolescencia. En A. Bilbao, \& I. Morlans, Subjetivación, Adolescencia, Institución: Psicopatología Clínica y Social (pp. 61-86). Santiago, Chile: LOM Impresiones.

Dolto, F. (1981). La dificultad de vivir 1: Familia y sentimientos. Barcelona, España: Gedisa.

Freud, A. (1976). Psicoanálisis del desarrollo del niño y el adolescente. Buenos Aires, Argentina: Paidos.

Freud, S. (2013). Tres ensayos para una teoría sexual. En Obras Completas (Tomo VII). Buenos Aires, Argentina: Amorrortu. (Trabajo original publicado en 1905).

Gutton, P. (1993). Lo puberal. Buenos Aires, Argentina: Amorrortu.

Houzel, D. (2004). Los retos de la parentalidad. En L. Solís-Ponton (Ed.), La parentalidad. Desafío para el tercer milenio (pp. 27-31). México, México D.F: Editorial Manual Moderno.

Marty, F. (2001). Les parents face au risque de la violence des enfants et des adolescents. Le Carnet PSY, 2001/4(64), 25- 33. doi: 10.3917/1cp.064.0025

Marty, F. (2009). El accionar psicótico en la adolescencia. En A. Bilbao, \& I. Morlans, Subjetivación, Adolescencia, Institución: Psicopatología Clínica y Social (pp. 87-120). Santiago, Chile: LOM Impresiones.

Marty, F., \& Missonnier, S. (2010). Adolescence et monde virtual. Études, 2010/11, TOM 413, 473-484.

Rev. Latinoam. Psicopat. Fund., São Paulo, 20(3), 451-464, set. 2017 


\section{ARTIGOS}

Mazet, P. (2004). Postfacio para la edición en francés. En L. Solís-Ponton, La parentalidad. Desafio para el tercer milenio (pp. 200-202). México, México D.F: Editorial Manual Moderno.

Wettengel, L. (2009). Trazando surcos: el trabajo de la parentalidad. En L. Wettengel, G. Untoiglich, \& G. Szyber, Patologías actuales en la infancia. Bordes y desbordes en clínica y educación (pp. 35-52). Buenos Aires, Argentina: Noveduc.

\section{Resúmenes}

( $\mathrm{O}$ exercício da parentalidade na clínica com adolescentes: uma proposta de intervenção psicanalítica)

Mediante a revisão de um caso clínico, procura-se analisar as configurações do exercício da parentalidade na clínica com adolescentes, desde uma perspectiva psicanalitica, a fim de propor a integração e análise nessas intervenções. Este trabalho não implicaria apenas transformações psíquicas para os adolescentes, mas também para os pais, aqueles que estão envolvidos em sua própria história.

Palavras-chave: Adolescência, parentalidade, psicologia clínica, psicanálise

(Parenting within clinical psychology with teenagers: a proposal of psychoanalytic intervention)

Through review of a clinical case, this paper analyzes the various configurations of parenting within clinical psychology with teenagers, from a psychoanalytic perspective, in order to propose integration and analysis in these interventions. This exercise would not only imply psychic transformations in teenagers, but also in parents, who are involved in their history.

Key words: Adolescence, parenting, clinical psychology, psychoanalysis

(L'exercice de la parentalité dans le travail clinique avec des adolescents: une proposition de thérapie psychanalytique)

En s'appuyant sur l'étude d'un cas clinique, il s'agit d'analyser d'un point de vue psychanalytique les configurations de l'exercice de la parentalité dans le travail clinique avec des adolescents, dans le but de proposer son intégration et son analyse dans ce contexte. Cet exercice suggèrerait des changements psychiques non seulement pour les adolescents mais aussi pour les parents, qui sont à leur tour impliqués de par leur propre histoire.

Mots clés: Adolescence, parentalité, psychologie clinique, psychanalyse 


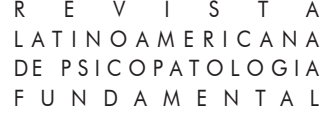

(Die Ausübung der Elternschaft in der Klinik von Jugendlichen: Vorschlag einer psychoanalytischen Intervention)

Basierend auf einem klinischen Fallbericht analysierten wir die Konfigurationen der Ausübung der Elternschaft in der Klinik von Jugendlichen aus psychoanalytischer Sicht, um ihre Integration und Analyse in diesen Interventionen vorzuschlagen. Der vorliegende Artikel betrachtet jedoch nicht nur die psychischen Veränderungen von Jugendlichen, sondern schließt ebenfalls die der Eltern ein, welche mit ihrer eigenen Geschichte konfrontiert werden.

Schlüsselwörter: Adoleszenz, Elternschaft, klinische Psychologie, Psychoanalyse

Citação/Citation: Olguín, D. F. (2017, setembro). El ejercício de la parentalidad en la clínica con adolescentes: una propuesta de intervencion psicoanalítica. Revista Latinoamericana de Psicopatologia Fundamental, 20(3), 451-464. http://dx.doi.org/10.1590/1415-4714.2017v20n3p451.3

Editores do artigo/Editors: Profa. Dra. Ana Maria Rudge e Profa. Dra. Sonia Leite. Recebido/Received: 18.7.2016/ 7.18.2016 Aceito/Accepted: 25.9.2016 / 9.25.2016

Copyright: (C) 2009 Associação Universitária de Pesquisa em Psicopatologia Fundamental/ University Association for Research in Fundamental Psychopathology. Este é um artigo de livre acesso, que permite uso irrestrito, distribuição e reprodução em qualquer meio, desde que o autor e a fonte sejam citados / This is an open-access article, which permits unrestricted use, distribution, and reproduction in any medium, provided the original authors and sources are credited.

Financiamento/Funding: A autora declara não ter sido financiada ou apoiada. The author has no support or funding funded to report.

Conflito de interesses/Conflict of interest: A autora declara que não há conflito de interesses / The author has no conflict of interest to declare.

\section{Daniela Fernández Olguín}

Psicóloga; Magister en Psicología Clínica con Niños y Jóvenes.

Universidad de Viña del Mar

Escuela de Ciencias Jurídicas y Sociales

Los Fresnos 91, Miraflores

Vinal del Mar, Chile Código Postal 2561091

daniela.paz.f@gmail.com

This is an open-access article, which permits unrestricted use, distribution, and reproduction in any medium for non-commercial purposes provided the original authors and sources are credited. 